\title{
Wavelet-Based Feature Extraction for the Analysis of EEG Signals Associated with Imagined Fists and Feet Movements
}

\author{
Mohammad H. Alomari ${ }^{1}$, Emad A. Awada ${ }^{1}$, Aya Samaha $^{1}$ \& Khaled Alkamha ${ }^{1}$ \\ ${ }^{1}$ Applied Science University, Amman, Jordan \\ Correspondence: Mohammad H. Alomari, Electrical and Computer Engineering Department, Faculty of \\ Engineering, Applied Science University, P.O. Box 166, Amman 11931, Jordan. Tel: 962-6560-9999 ext. 1434. \\ E-mail: m_alomari@asu.edu.jo
}

Received: December 21, 2013 Accepted: February 7, 2014 Online Published: March 2, 2014

doi:10.5539/cis.v7n2p17

URL: http://dx.doi.org/10.5539/cis.v7n2p17

\begin{abstract}
Electroencephalography (EEG) signals were analyzed in many research applications as a channel of communication between humans and computers. EEG signals associated with imagined fists and feet movements were filtered and processed using wavelet transform analysis for feature extraction. The proposed work used Neural Networks (NNs) as a classifier that enables the classification of imagined movements into either fists or feet. Wavelet families such as Daubechies, Symlets, and Coiflets wavelets were used to analyze the extracted events and then different feature extraction measures were calculated for three detail levels of the wavelet coefficients. Intensive NN training and testing experiments were carried out and different network configurations were compared. The optimum classification performance of $89.11 \%$ was achieved with a NN classifier of 20 hidden layers while using the Mean Absolute Value (MAV) of the Coiflets wavelet coefficients as inputs to NN. The proposed system showed a good performance that enables controlling computer applications via imagined fists and feet movements.
\end{abstract}

Keywords: Discrete Wavelet Transform (DWT), Electroencephalography (EEG), Brain-Computer Interface (BCI), machine learning, Neural Networks (NN), feature extraction, data mining

\section{Introduction}

Electroencephalography (EEG) is defined as the process of measuring the electrical voltage fluctuations along the scalp as a result of the current flows in brain's neurons and the brain's neural activity (Niedermeyer \& da Silva, 2005). In typical EEG tests the brain's electrical activity is monitored and recorded using electrodes that are fixed on the scalp (Sleight et al., 2009). Brain-Computer Interface (BCI) is a combination of hardware and software systems that enables the use of the brain's neural activity to communicate with others or to control machines, artificial limbs, or robots without direct physical movements (Levine et al., 1999; Donoghue, 2002; Wolpaw et al., 2002; Vallabhaneni et al., 2005). BCI captures EEG signals in conjunction with a specific user activity then uses different signal processing algorithms to translate these records into control commands for different machine and computer applications (Graimann et al., 2010). It is proved in (Vidal, 1973) that a user's intent could be effectively represented by signals recorded from brain activity.

During the last few years, BCI has become an attractive field of research and applications specially in helping disabled individuals by providing a new channel of communication with the external environment and offering a feasible tool to control artificial limbs (Selim et al., 2008). A variety of BCI applications were described in (Grabianowski, 2007). BCI is a highly interdisciplinary research topic that combines medicine, neurology, psychology, rehabilitation engineering, Human-Computer Interaction ( $\mathrm{HCI})$, signal processing and machine learning (Smith et al., 2007).

It can be noted from the literature that the strength of any BCI application depends on the translation approach used to transform EEG signal patterns into machine commands. In (Pfurtscheller et al., 1997), the authors recorded EEG signals for three subjects while imagining either right or left hand movement based on a visual cue stimulus. They were able to classify EEG signals into right and left hand movements using a neural network classifier with an accuracy of $80 \%$ and concluded that this accuracy did not improve with increasing number of sessions. Sepulveda (2011) used features produced by Motor Imagery (MI) to control a robot arm. Features such as the band power in specific frequency bands (alpha: 8-12 Hz and beta: 13-30 Hz) were mapped into right and 
left limb movements. In addition, they used similar features with MI, which are the Event Related Desynchronization and Synchronization (ERD/ERS) comparing the signal's energy in specific frequency bands with respect to the mentally relaxed state.

It was shown in (Mohamed, 2011; Alomari et al., 2013) that the combination of ERD/ERS and Movement-Related Cortical Potentials (MRCP) improves EEG classification as this offers an independent and complimentary information. The authors of (Farina et al., 2007) presented an approach for the classification of single trial MRCP using a discrete dyadic wavelet transform and Support Vector Machines (SVMs) and they provided a promising classification performance. A single trial right/left hand movement classification is reported in (Kim et al., 2003). The authors analyzed both executed and imagined hand movement EEG signals and created a feature vector consisting of the ERD/ERS patterns of the mu and beta rhythms and the coefficients of the autoregressive model. Artificial Neural Networks (ANNs) is applied to two kinds of testing datasets and an average recognition rate of $93 \%$ is achieved.

A three-class BCI system was presented in (Wang et al., 2007) for the translation of imagined left/right hands and foot movements into commands that operates a wheelchair. This work used many spatial patterns of ERD on $\mathrm{mu}$ rhythms along the sensory-motor cortex and the resulting classification accuracy for online and offline tests was $79.48 \%$ and $85.00 \%$, respectively. The authors of (Guger et al., 1999) proposed an EEG-based BCI system that controls hand prosthesis of paralyzed people by movement thoughts of left and right hands. They reported an accuracy of about $90 \%$.

In Su et al. (2011), a hybrid BCI control strategy is presented. The authors expanded the control functions of a P300 potential based BCI for virtual devices and MI related sensorimotor rhythms to navigate in a virtual environment. Imagined left/right hand movements were translated into movement commands in a virtual apartment and an extremely high testing accuracy results were reached.

Homri et al. (2012) applied the Daubechies, Coiflet and Symmlet wavelet families to a dataset of MI to extract features and describe right and left hand movement imagery. The authors reported that the use of Linear Discriminate Analysis (LDA) and Multilayer Perceptron (MLP) Neural Networks (NNs) provided good classification results and that LDA classifier achieved higher classification results of up to $88 \%$ for different Symmlet wavelets. Tolić and Jović (2013) used the discrete wavelet transform (DWT) to create inputs for a NNs classifier and the authors reported a very high classification accuracy of $99.87 \%$ for the recognition of some mental tasks.

We proposed a system that could efficiently discriminate between executed left and right fist movements in our previous study (Alomari et al., 2013). The current work is an extension for our studies to classify both imagined fists and feet movements by analyzing EEG signals recorded during a large number of experiments for 100 different subjects. Many wavelet families were used to calculate wavelet coefficients and then all the possible feature candidates were extracted and used in the training/testing and optimization experiments of a NNs classifier.

\section{The Physionet EEG Dataset}

In this work, we used the EEG dataset that was created and contributed to PhysioNet (Goldberger et al., 2000) by the developers of the BCI2000 (Schalk et al., 2004) instrumentation system. The dataset is publically available online at http://www.physionet.org/pn4/eegmmidb. It consists of more than 1500 one or two minutes-duration EEG records obtained from 109 healthy subjects. Subjects were asked to execute and imagine different tasks while 64 channels of EEG signals were recorded from the electrodes that were fitted along the scalp.

In the records of the dataset that are related to the current research, each subject performed three experimental runs of imagining the movement of both fists or both feet. During each two-minute run, either the top or the bottom of a computer screen shows a target. The subject imagines opening and closing either both fists (if the target is on top) or both feet (if the target is on the bottom) until the target disappears where he relaxes. This was repeated 15 times during each two-minute run. Then the obtained EEG signals were recorded according to the international 10-20 system as seen in Figure 1. For this work, we created a subset of three two-minute runs for 100 subjects for a total of 4500 events (45 imagined events per subject). 

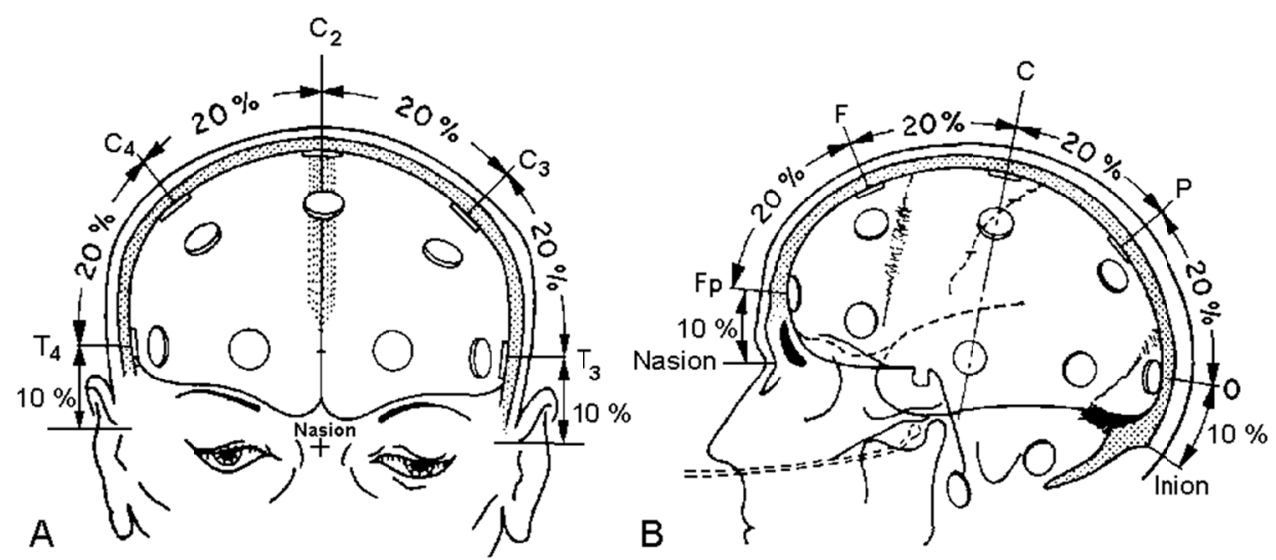

Figure 1. The International 10-20 system (Jasper, 1958) as seen from (A) front and (B) left of the head

\section{Preprocessing}

\subsection{Channel Selection}

It was shown in the literature that most of the EEG channels are representing redundant information (Sleight et al., 2009). In addition, it was concluded that the neural activity that is mostly correlated to the fists movements is almost exclusively contained within the channels $\mathrm{C}_{3}, \mathrm{C}_{4}$, and $\mathrm{C}_{\mathrm{Z}}$ of the EEG channels as depicted in Figure 1.A (Deecke et al., 1982; Neuper \& Pfurtscheller, 2001). So, we assumed that there is no need to analyze all the available EEG channels and it is more efficient to process the $\mathrm{C}_{3}, \mathrm{C}_{4}$, and $\mathrm{C}_{Z}$ channels of data.

\subsection{Filtering}

EEGs are noisy and non-stationary signals that have to be filtered to get rid of the unnecessary content from the raw signals (Romo-Vazquez et al., 2007). As shown in Figure 2, the first step of the proposed system is to filter the selected channels for the purpose of removing the DC (direct current) shifts and minimizing the presence of filtering artifacts at epoch boundaries. This was accomplished by applying a band pass filter from $0.5 \mathrm{~Hz}$ to 50 $\mathrm{Hz}$ using the interactive MATLAB toolbox EEGLAB (Delorme \& Makeig, 2004).

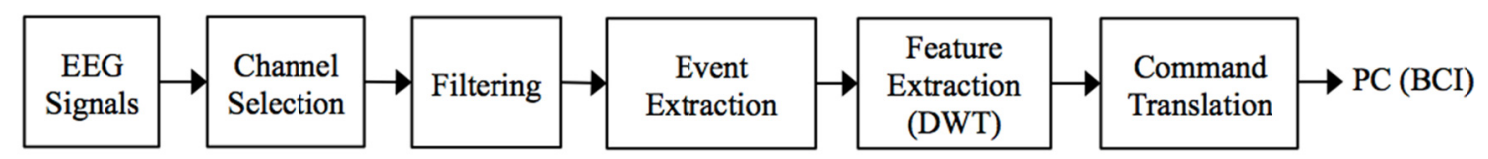

Figure 2. A block diagram of the proposed system

It is shown in (Bartels et al., 2010) that EEG signals are usually masked by physiological artifacts that produce huge amounts of useless data. Eye and muscle movements could be good examples of these artifacts that constitute a challenge in the field of BCI research. Automatic Artifact Removal (AAR) automatically removes artifacts from EEG data based on blind source separation and other various algorithms. The AAR toolbox (Gómez-Herrero, 2008) was implemented as an EEGLAB plug-in and was used to process our EEG data subset on two stages: Electrooculography (EOG) removal using the Blind Source Separation (BSS) algorithm then Electromyography (EMG) Removal using the same algorithm (Joyce et al., 2004).

\subsection{Event Splitting}

As described previously, each two-minute EEG record includes 15 events that are separated by a short neutral period where the subject relaxes. A subject imagines opening and closing his fists or feet and keeps doing this for 4.1 seconds then he takes a rest for the duration of 4.2 seconds. These events count up to a sum of 120.3 seconds representing the two-minute record. In our experiments, data that are time locked to a specific event type were split in a separate vector. As the Physionet EEG dataset was sampled at 160 samples per second, each vector includes 656 samples (values) of the original recorded EEG signal. 


\section{Wavelet Transform Analysis}

\subsection{Wavelet Analysis for EEG Signals}

The Wavelet transform analysis was used in a wide range of research topics within the field of signal processing (Riouel \& Vetterli, 1991; Silverman, 2000; Oliver et al., 2005; Phinyomark et al., 2011, 2012; Awada \& Alomari, 2013). Based on a multi-resolutions process, the wavelet properties of a scalable window allow pinpointing signal components. These properties of dilation and translation enable the extraction of all components for every position by creating different scales and shifted functions (in time domain) of a signal (Tuntisak \& Premrudeepreechacharn, 2007; Qingyang \& Zhe, 2012). As a result, wavelet finer and large scaling, permit all information of the signal (the big picture), while small scales shows signal details by zooming into the signal components.

For discrete data, such as the datasets used in the current work, the Discrete Wavelet Transform (DWT) is better fit for analysis. It was shown in (Phinyomark et al., 2010) that a suitable wavelet function must be used to optimize the analysis performance. A large selection of DWT mother wavelets is available to be used in our work. But the Daubechies (Db), Symlets (Sym), and Coiflets (Coif) wavelets were proved to be the most suitable families in similar applications (Mahaphonchaikul et al., 2010; Phinyomark et al., 2010; Homri et al., 2012; Kharat \& Dudul, 2012; Michahial et al., 2012). So, in this work it was decided to calculate the Daubechies orthogonal wavelets Db2-Db20 (even indices), the Symlets wavelets Sym2-Sym20 (even indices), and the Coiflets wavelets Coif1-Coif5.

As shown in Figure 3, the main purpose of the DWT is to decompose the recorded EEG signal into multi-resolution subsets of coefficients: a detailed coefficient subset $\left(\mathrm{cD}_{i}\right)$ and an approximation coefficient subset $\left(\mathrm{cA}_{i}\right)$ at the level $i$. So, at the first decomposition level we obtain $\mathrm{cD}_{1}$ and $\mathrm{cA}_{1}$ then the first approximation $\mathrm{cA}_{1}$ can be transformed into $\mathrm{cD}_{2}$ and $\mathrm{cA}_{2}$ at the second level, and so on. For our experiments, the decomposition level was set to generate four level details.

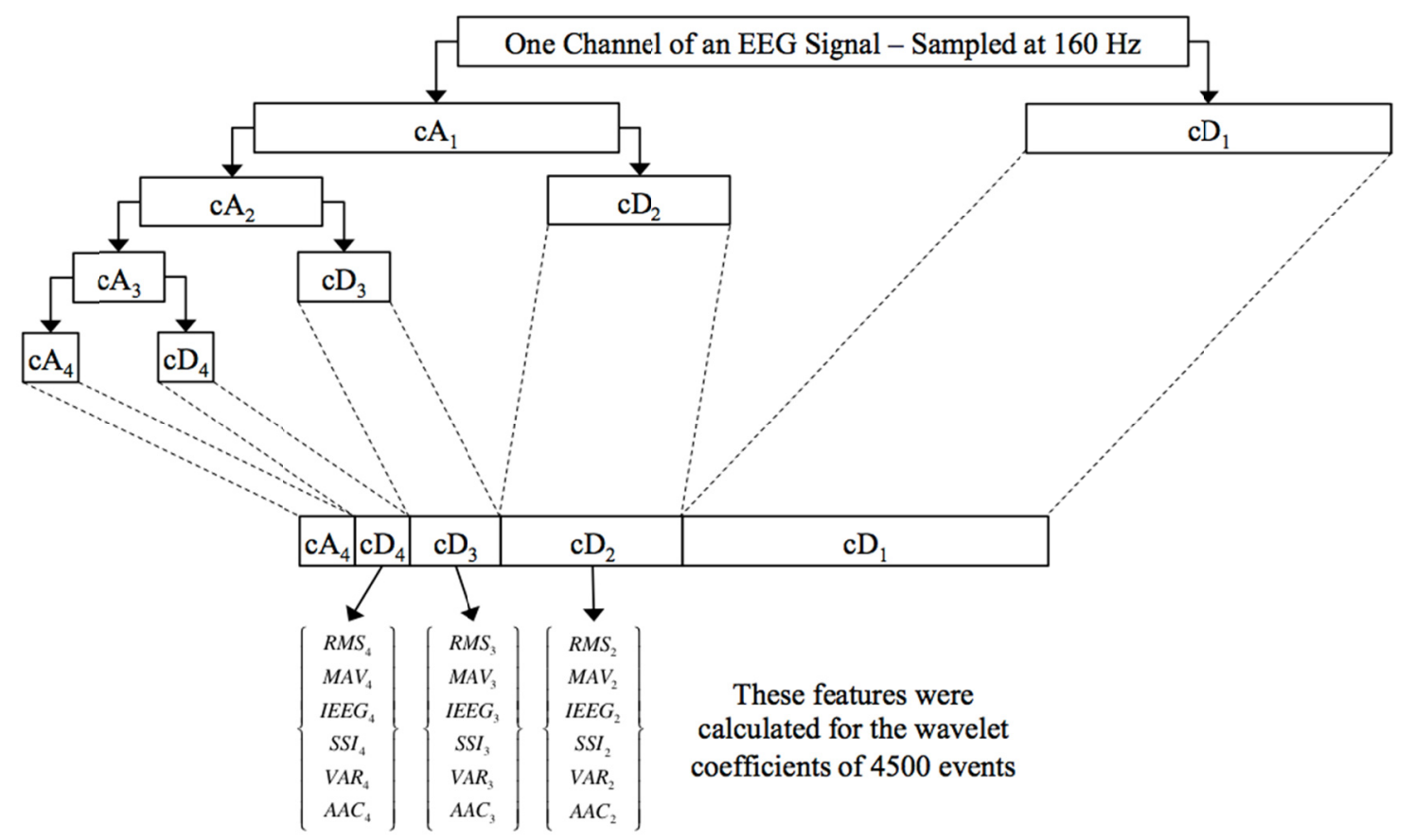

Figure 3. Feature Extraction by the multi-resolution decomposition of EEG signals

\subsection{Feature Vectors Construction}

The wavelet transformation of any EEG record at four levels results in four details and one approximation with the frequency ranges listed in Table 1. There are many electrophysiological features that are associated with the brain's normal motor output channels (Wolpaw et al., 2002; Bashashati et al., 2007). Some of these important features are the $\mathrm{mu}(8-12 \mathrm{~Hz})$ and beta $(13-30 \mathrm{~Hz})$ rhythms (Alomari et al., 2013). We concluded from Table 1 that the details $\mathrm{cD}_{2}, \mathrm{cD}_{3}$, and $\mathrm{cD}_{4}$ provide proper representation for the mu and beta rhythms and we decided to 
extract the vectors of features from these details only as shown in Figure 3.

Table 1. Frequency range for the decomposed details and approximation

\begin{tabular}{cc}
\hline Signal Component & Frequency Range \\
\hline $\mathrm{cD}_{1}$ & $40-80 \mathrm{~Hz}$ \\
$\mathrm{cD}_{2}$ & $20-40 \mathrm{~Hz}$ \\
$\mathrm{cD}_{3}$ & $10-20 \mathrm{~Hz}$ \\
$\mathrm{cD}_{4}$ & $5-10 \mathrm{~Hz}$ \\
$\mathrm{cA}_{4}$ & $0-5 \mathrm{~Hz}$ \\
\hline
\end{tabular}

Phinyomark et al. (2013) provided the mathematical definitions of many amplitude estimators for neurological activities. If we assume that the $n^{\text {th }}$ sample of a wavelet decomposed detail at level $i$ is $D_{i}(n)$, then we can define the following features:

- $\quad$ Root Mean Square (RMS)

$$
R M S_{i}=\sqrt{\frac{1}{N} \sum_{n=1}^{N} D_{i}^{2}(n)}
$$

- Mean Absolute Value (MAV)

$$
M A V_{i}=\frac{1}{N} \sum_{n=1}^{N}\left|D_{i}(n)\right|
$$

- Integrated EEG (IEEG)

$$
\text { IEE } G_{i}=\sum_{n=1}^{N}\left|D_{i}(n)\right|
$$

- $\quad$ Simple Square Integral (SSI)

$$
S S I_{i}=\sum_{n=1}^{N}\left|D_{i}(n)\right|^{2}
$$

- Variance of EEG (VAR)

$$
V A R_{i}=\frac{1}{N-1} \sum_{n=1}^{N} D_{i}^{2}(n)
$$

- $\quad$ Average Amplitude Change (AAC)

$$
A A C_{i}=\frac{1}{N} \sum_{n=1}^{N}\left|D_{i}(n+1)-D_{i}(n)\right|
$$

The Daubechies, Symlets, and Coiflets wavelets were used to analyze the channels $C_{3}, C_{4}$, and $C_{z}$ of each EEG record. Then, as depicted in Figure 3, the features RMS, MAV, IEEG, SSI, VAR, and AAC were calculated for the wavelet coefficients using Equations 1 through 6 . This process was repeated for each event in our dataset of 4500 vectors. At the end of these calculations, 9 RMS features ( 3 channels $\times 3$ details), 9 MAV features, 9 IEEG features, 9 SSI features, 9 VAR features, and 9 AAC features were generated for each wavelet. These features were numerically represented in a format that is suitable for use with NN algorithms (Qahwaji et al., 2008; Al-Omari et al., 2010) as described in the next section.

\section{Neural Networks Experiments}

Neural networks learning algorithms were used in (Pfurtscheller et al., 1997; Homri et al., 2012; Kharat \& Dudul, 2012; Tolić \& Jović, 2013) and provided good classification performance. A detailed description of NN can be found in (Qahwaji et al., 2008). The MATLAB NN toolbox was used for all the training and testing experiments. The training experiments were handled with the aid of the back-propagation learning algorithm (Fahlmann \& Lebiere, 1989). 
Six networks were created with one output node representing the target function of fists/feet movement:

- Network 1:9 inputs representing the MAR Features.

- Network 2: 9 inputs representing the RMS Features.

- Network 3: 9 inputs representing the AAC Features.

- Network 4: 9 inputs representing the IEEG Features.

- Network 5: 9 inputs representing the SSI Features.

- Network 6: 9 inputs representing the VAR Features.

The number of hidden layers for these networks was varied from 1 to 20 . At each specific number of hidden layers, $80 \%$ of the samples (3600 events) were randomly selected and used for training and the remaining $20 \%$ for testing. This process was repeated 10 times, and in each time the datasets were randomly mixed. For each number of hidden layers, the average accuracy was calculated for the ten training-testing pairs.

A huge number of training and testing experiments were carried out for each of the six networks. The performance of each network, for a specific wavelet family, is optimized by plotting all classification results on the same graph as shown in Figure 4. Table 3 through Table 4 lists the number of hidden layers associated with the neural network that provided the best average accuracy, for a specific wavelet function.

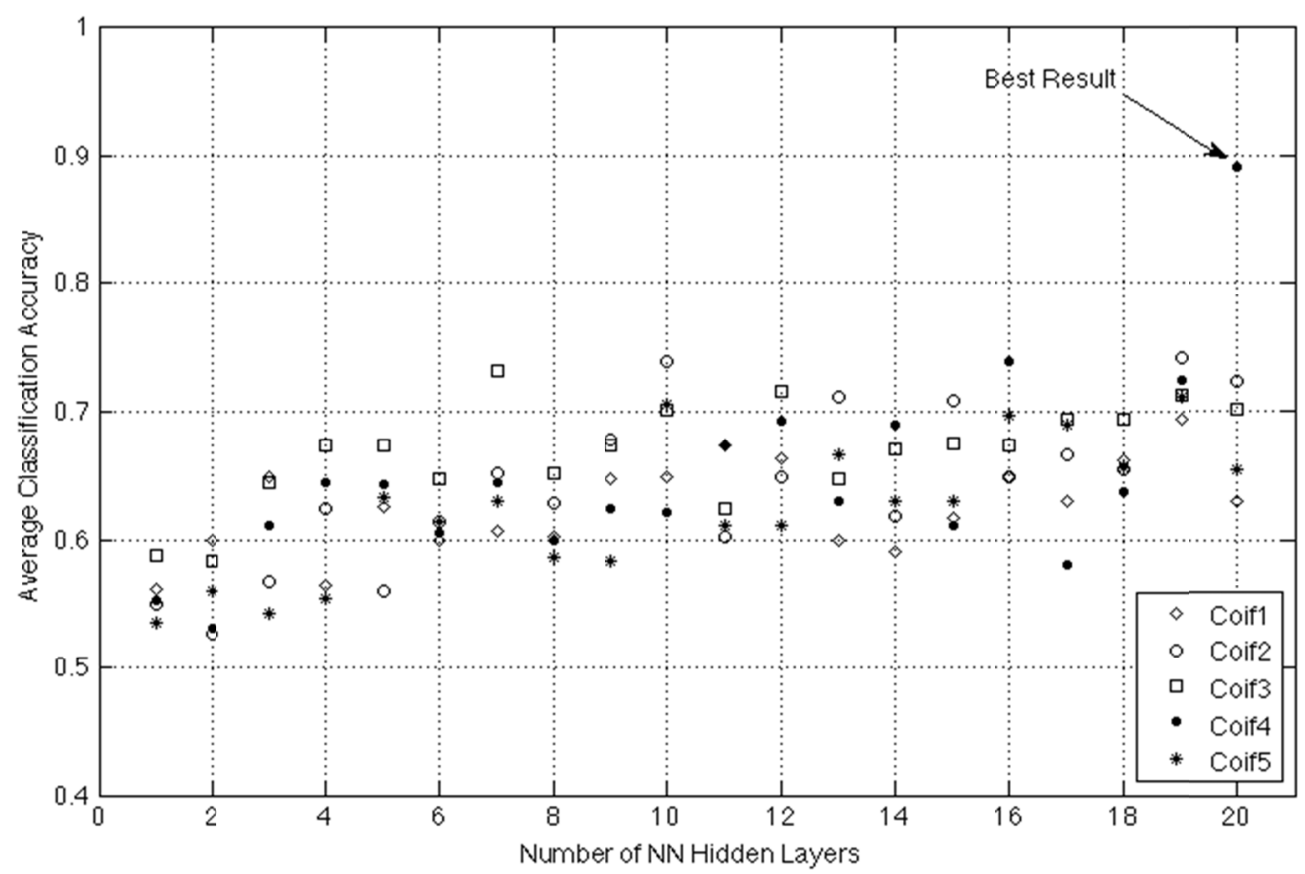

Figure 4. Optimization graph comparing the NN performance using the MAV feature for all Coiflets functions

Table 2. Best Average Accuracy (Avg Acc) results achieved using different Coiflets functions with different features and a variable number of Hidden Layers (HL) for the NN classifier

\begin{tabular}{cccccccccccccc}
\hline Features & \multicolumn{2}{c}{ MAV } & \multicolumn{2}{c}{ RMS } & \multicolumn{2}{c}{ AAC } & \multicolumn{2}{c}{ IEEG } & \multicolumn{2}{c}{ SSI } & \multicolumn{2}{c}{ VAR } \\
\hline $\begin{array}{c}\text { Coiflets } \\
\text { wavelet }\end{array}$ & HL & $\begin{array}{c}\text { Avg } \\
\text { Acc }\end{array}$ & HL & $\begin{array}{c}\text { Avg } \\
\text { Acc }\end{array}$ & HL & $\begin{array}{c}\text { Avg } \\
\text { Acc }\end{array}$ & HL & $\begin{array}{c}\text { Avg } \\
\text { Acc }\end{array}$ & HL & $\begin{array}{c}\text { Avg } \\
\text { Acc }\end{array}$ & $\begin{array}{c}\text { HL } \\
\text { Avg } \\
\text { Acc }\end{array}$ \\
\hline Coif1 & 19 & 0.6945 & 11 & 0.7537 & 9 & 0.7395 & 5 & 0.7205 & 14 & 0.7348 & 16 & 0.7561 \\
Coif2 & 19 & 0.7419 & 13 & 0.6874 & 16 & 0.7419 & 20 & 0.7892 & 19 & 0.7348 & 6 & 0.6495 \\
Coif3 & 7 & 0.7324 & 17 & 0.7656 & 18 & 0.7395 & 11 & 0.7490 & 20 & 0.7040 & 15 & 0.7158 \\
Coif4 & 20 & 0.8911 & 19 & 0.7442 & 16 & 0.7916 & 12 & 0.7277 & 18 & 0.7111 & 17 & 0.7845 \\
Coif5 & 19 & 0.7111 & 14 & 0.7324 & 19 & 0.7750 & 11 & 0.7111 & 19 & 0.6684 & 15 & 0.6874 \\
\hline
\end{tabular}


Table 3. Best Average Accuracy (Avg Acc) results achieved using different Daubechies functions with different features and a variable number of Hidden Layers (HL) for the NN classifier

\begin{tabular}{cccccccccccccc}
\hline Features & \multicolumn{2}{c}{ MAV } & \multicolumn{2}{c}{ RMS } & \multicolumn{2}{c}{ AAC } & \multicolumn{2}{c}{ IEEG } & \multicolumn{2}{c}{ SSI } & \multicolumn{3}{c}{ VAR } \\
\hline $\begin{array}{c}\text { Daubechies } \\
\text { wavelet }\end{array}$ & HL & $\begin{array}{c}\text { Avg } \\
\text { Acc }\end{array}$ & HL & $\begin{array}{c}\text { Avg } \\
\text { Acc }\end{array}$ & HL & $\begin{array}{c}\text { Avg } \\
\text { Acc }\end{array}$ & HL & $\begin{array}{c}\text { Avg } \\
\text { Acc }\end{array}$ & HL & $\begin{array}{c}\text { Avg } \\
\text { Acc }\end{array}$ & $\begin{array}{c}\text { HL } \\
\text { Avg } \\
\text { Acc }\end{array}$ \\
\hline Db2 & 17 & 0.8403 & 12 & 0.8203 & 20 & 0.8028 & 19 & 0.8003 & 15 & 0.8478 & 19 & 0.8028 \\
Db4 & 11 & 0.8378 & 18 & 0.8053 & 18 & 0.7628 & 20 & 0.8478 & 8 & 0.7278 & 17 & 0.7528 \\
Db6 & 17 & 0.7678 & 17 & 0.7178 & 11 & 0.7578 & 15 & 0.7503 & 15 & 0.8328 & 10 & 0.7153 \\
Db8 & 8 & 0.8103 & 20 & 0.7753 & 17 & 0.7353 & 17 & 0.8678 & 14 & 0.7353 & 20 & 0.7978 \\
Db10 & 12 & 0.7853 & 11 & 0.8078 & 14 & 0.8228 & 16 & 0.8353 & 12 & 0.6803 & 9 & 0.6903 \\
Db12 & 17 & 0.7678 & 8 & 0.7228 & 18 & 0.7653 & 19 & 0.7578 & 18 & 0.7203 & 18 & 0.7128 \\
Db14 & 20 & 0.7503 & 13 & 0.7728 & 13 & 0.7678 & 12 & 0.8303 & 17 & 0.7128 & 18 & 0.7353 \\
Db16 & 16 & 0.8178 & 13 & 0.7453 & 19 & 0.7753 & 14 & 0.7178 & 18 & 0.7128 & 12 & 0.7153 \\
Db18 & 16 & 0.8028 & 16 & 0.7303 & 20 & 0.7203 & 18 & 0.7328 & 10 & 0.7303 & 20 & 0.7278 \\
Db20 & 14 & 0.7803 & 15 & 0.8153 & 15 & 0.7478 & 12 & 0.7703 & 17 & 0.6978 & 17 & 0.8003 \\
\hline
\end{tabular}

Table 4. Best Average Accuracy (Avg Acc) results achieved using different Symlets functions with different features and a variable number of Hidden Layers (HL) for the NN classifier

\begin{tabular}{cccccccccccccc}
\hline Features & \multicolumn{2}{c}{ MAV } & \multicolumn{2}{c}{ RMS } & \multicolumn{2}{c}{ AAC } & \multicolumn{2}{c}{ IEEG } & \multicolumn{3}{c}{ SSI } & \multicolumn{3}{c}{ VAR } \\
\hline $\begin{array}{c}\text { Symlets } \\
\text { wavelet }\end{array}$ & HL & $\begin{array}{c}\text { Avg } \\
\text { Acc }\end{array}$ & HL & $\begin{array}{c}\text { Avg } \\
\text { Acc }\end{array}$ & HL & $\begin{array}{c}\text { Avg } \\
\text { Acc }\end{array}$ & HL & $\begin{array}{c}\text { Avg } \\
\text { Acc }\end{array}$ & HL & $\begin{array}{c}\text { Avg } \\
\text { Acc }\end{array}$ & $\begin{array}{c}\text { HL } \\
\text { Avg } \\
\text { Acc }\end{array}$ \\
\hline Sym2 & 16 & 0.8451 & 14 & 0.8378 & 17 & 0.8549 & 17 & 0.8256 & 20 & 0.7620 & 14 & 0.8744 \\
Sym4 & 20 & 0.7522 & 14 & 0.7644 & 16 & 0.7816 & 18 & 0.7669 & 14 & 0.6789 & 19 & 0.7278 \\
Sym6 & 14 & 0.7596 & 13 & 0.7840 & 14 & 0.8036 & 9 & 0.7889 & 18 & 0.6764 & 19 & 0.6838 \\
Sym8 & 15 & 0.6984 & 17 & 0.7009 & 15 & 0.7473 & 16 & 0.7596 & 10 & 0.6936 & 19 & 0.7107 \\
Sym10 & 13 & 0.7180 & 8 & 0.7009 & 16 & 0.8500 & 15 & 0.7473 & 16 & 0.6911 & 20 & 0.6984 \\
Sym12 & 19 & 0.7718 & 14 & 0.7473 & 20 & 0.7424 & 18 & 0.7156 & 11 & 0.7449 & 13 & 0.6838 \\
Sym14 & 18 & 0.8084 & 11 & 0.7449 & 19 & 0.7889 & 20 & 0.7840 & 19 & 0.7180 & 18 & 0.6960 \\
Sym16 & 19 & 0.7327 & 14 & 0.7498 & 16 & 0.7816 & 18 & 0.7596 & 17 & 0.6667 & 20 & 0.6740 \\
Sym18 & 14 & 0.7327 & 18 & 0.8231 & 11 & 0.8182 & 20 & 0.7547 & 16 & 0.7204 & 19 & 0.7204 \\
Sym20 & 19 & 0.7376 & 16 & 0.7522 & 19 & 0.8427 & 19 & 0.7669 & 15 & 0.7204 & 15 & 0.6862 \\
\hline
\end{tabular}

By comparing the results, it was found that the optimum classification accuracy that can be achieved using our system is $89.11 \%$. This performance was achieved by inputting the MAV feature of a Coif4 wavelet into a neural network of 20 hidden layers. This result is consistent with the conclusions reported in (Phinyomark et al., 2013) where it was shown that both MAV and RMS were accurate inputs for recognition and classification systems.

If we compare the highest accuracies in all tables, we can note that the Symlets wavelet outperforms the other wavelet families in most cases. The VAR feature for the Sym2 wavelet provided an accuracy of $87.44 \%$ using a $\mathrm{NN}$ of 14 hidden layers. In addition, the AAC feature for the same wavelet provided a $85.49 \%$ performance using a $\mathrm{NN}$ of 17 hidden layers.

On the other hand, it can be concluded from all tables that the MAV feature provides the best overall performance using any wavelet family. It is associated with the performances of $89.11 \%, 84.03 \%$, and 84.51 while applying the Coiflets, Daubechies, and Symlets, respectively. The next best feature is the VAR then the IEEG. It can be mentioned here that The IEEG feature for the Db8 wavelet provided an accuracy of $86.78 \%$ using a NN of 17 hidden layers. 


\section{Conclusions}

This work describes a classification system that can classify imagined EEG signals into fists and feet movements. Symlets, Daubechies, Coiflets wavelet families were compared for their abilities to decompose EEG signals and extract features that can be used as inputs to neural networks. Extensive experiments were carried out and the neural networks were optimized. The optimum classification performance of $89.11 \%$ was achieved with a NN classifier of 20 hidden layers while using the mean absolute value of the Coiflets wavelet coefficients as inputs to NN. It is believed that this work is one of the best to achieve such classification performance while working on imagined fists and feet activities. Real-time applications of this work can be implemented in the near future.

\section{Acknowledgements}

The authors are grateful to Applied Science University (ASU), Amman-Jordan, for the financial support granted to cover the publication fee of this research article.

\section{References}

Al-Omari, M., Qahwaji, R., Colak, T., \& Ipson, S. (2010). Machine learning-based investigation of the associations between cmes and filaments. Solar Physics, 262(2), 511-539. http://dx.doi.org/10.1007/s11207-010-9516-5

Alomari, M. H., Samaha, A., \& AlKamha, K. (2013). Automated Classification of L/R Hand Movement EEG Signals using Advanced Feature Extraction and Machine Learning. International Journal of Advanced Computer Science and Applications, 4(6), 207-212. http://dx.doi.org/10.14569/IJACSA.2013.040628

Awada, E. A., \& Alomari, M. H. (2013). Application of Wavelet Transform Analysis to ADCs Harmonics Distortion. Computer and Information Science, 6(3), 118-124. http://dx.doi.org/10.5539/cis.v6n3p118

Bartels, G., Shi, L. C., \& Lu, B. L. (2010). Automatic artifact removal from EEG - a mixed approach based on double blind source separation and support vector machine. 2010 Annual International Conference of the IEEE Engineering in Medicine and Biology Society (EMBC) (pp. 5383-5386). http://dx.doi.org/10.1109/iembs.2010.5626481

Bashashati, A., Fatourechi, M., Ward, R. K., \& Birch, G. E. (2007). A survey of signal processing algorithms in brain-computer interfaces based on electrical brain signals. Journal of Neural Engineering, 4(2), R32-57. http://dx.doi.org/10.1088/ 1741-2560/4/2/R03

Deecke, L., Weinberg, H., \& Brickett, P. (1982). Magnetic fields of the human brain accompanying voluntary movements: Bereitschaftsmagnetfeld. Experimental Brain Research, 48(1), 144-148. http://dx.doi.org/10.1007/ BF00239582

Delorme, A., \& Makeig, S. (2004). EEGLAB: an open source toolbox for analysis of single-trial EEG dynamics. Journal of Neuroscience Methods, 134(1), 9-21. http://dx.doi.org/10.1016/ j.jneumeth.2003.10.009

Donoghue, J. P. (2002). Connecting cortex to machines: recent advances in brain interfaces. Nature Neuroscience Supplement, 5(Suppl.), 1085-1088. http://dx.doi.org/10.1038/nn947

Fahlmann, S. E., \& Lebiere, C. (1989). The cascade-correlation learning architecture. Advances in Neural Information Processing Systems 2 (NIPS-2). Denver, Colorado.

Farina, D., Nascimento, O. F. D., Lucas, M. F., \& Doncarli, C. (2007). Optimization of wavelets for classification of movement-related cortical potentials generated by variation of force-related parameters. Journal of Neuroscience Methods, 162(1-2), 357-363. http://dx.doi.org/10.1016/j.jneumeth.2007.01.011

Goldberger, A. L., Amaral, L. A., Glass, L., Hausdorff, J. M., Ivanov, P. C., Mark, R. G., ... Stanley, H. E. (2000). PhysioBank, PhysioToolkit, and PhysioNet: Components of a New Research Resource for Complex Physiologic Signals. Circulation, 101(23), e215-e220. http://dx.doi.org/ 10.1161/01.CIR.101.23.e215

Gómez-Herrero, G. (2008). Automatic Artifact Removal (AAR) toolbox for MATLAB. Transform methods for Electroencephalography (EEG). Retrieved from http://kasku.org/projects/eeg/aar.htm

Grabianowski, E. (2007). How Brain-computer Interfaces Work. HowStuffWorks, Inc. Retrieved from http://computer.howstuffworks.com/brain-computer-interface.htm

Graimann, B., Allison, B., \& Pfurtscheller, G. (2010). Brain-Computer Interfaces: A Gentle Introduction (pp. 1-27). Berlin Heidelberg: Brain-Computer Interfaces, Springer. http://dx.doi.org/10.1007/978-3-642-02091-9_1

Guger, C., Harkam, W., Hertnaes, C., \& Pfurtscheller, G. (1999). Prosthetic Control by an EEG-based Brain- 
Computer Interface (BCI). AAATE 5th European Conference for the Advancement of Assistive Technology, Düsseldorf, Germany. Retrieved from http://www.gtec.at/research/Publications/aaate.pdf

Homri, I., Yacoub, S., \& Ellouze, N. (2012). Optimal segments selection for EEG classification. 6th International Conference on Sciences of Electronics, Technologies of Information and Telecommunications (SETIT) (pp. 817-821), Sousse, Tunisia. http://dx.doi.org/10.1109/SETIT.2012.6482020

Jasper, H. H. (1958). The ten twenty electrode system of the international federation. Electroencephalography and Clinical Neurophysiology, 10, 371-375. http://ci.nii.ac.jp/naid/10020218106/

Joyce, C. A., Gorodnitsky, I. F., \& Kutas, M. (2004). Automatic removal of eye movement and blink artifacts from EEG data using blind component separation. Psychophysiology, 41(2), 313-325. http://dx.doi.org/10.1111/j.1469-8986.2003.00141.x

Kharat, P. A., \& Dudul, S. V. (2012). Daubechies Wavelet Neural Network Classifier for the Diagnosis of Epilepsy. Wseas Transactions on Biology and Biomedicine, 9(4), 103-113.

Kim, J. A., Hwang, D. U., Cho, S. Y., \& Han, S. K. (2003). Single trial discrimination between right and left hand movement with EEG signal. Proceedings of the 25th Annual International Conference of the IEEE Engineering in Medicine and Biology Society (pp. 3321-3324). Cancun, Mexico, Vol. 3324. http://dx.doi.org/10.1109/iembs.2003.1280855

Levine, S. P., Huggins, J. E., BeMent, S. L., Kushwaha, R. K., Schuh, L. A., Passaro, E. A., ... Ross, D. A. (1999). Identification of electrocorticogram patterns as the basis for a direct brain interface. Journal of Clinical Neurophysiology, 16(5), 439-447. http://dx.doi.org/10.1097/00004691- 199909000-00005

Mahaphonchaikul, K., Sueaseenak, D., Pintavirooj, C., Sangworasil, M., \& Tungjitkusolmun, S. (2010). EMG signal feature extraction based on wavelet transform. 2010 International Conference on Electrical Engineering/Electronics Computer Telecommunications and Information Technology (ECTI-CON), Chiang Mai (pp. 327-331). Retrieved from http://ieeexplore.ieee.org/xpls/ abs_all.jsp?arnumber $=5491475$

Michahial, S., Ranjith, K. R., Hemath, K. P., \& Puneeth, K. A. (2012). Hand rotate EEG signal feature extraction by second order Daubechies wavelet transform (DWT). 2012 Third International Conference on Computing Communication \& Networking Technologies (ICCCNT), Coimbatore (pp. 1-6). http://dx.doi.org/10.1109/ICCCNT.2012.6396080

Mohamed, A. K. (2011). Towards improved EEG interpretation in a sensorimotor BCI for the control of a prosthetic or orthotic hand. Faculty of Engineering. Johannesburg, University of Witwatersrand. Thesis: Master of Science in Engineering. Retrieved from http://hdl.handle.net/10539/10546

Müller, K. R., Krauledat, M., Dornhege, G., Curio, G., \& Blankertz, B. (2007). Machine learning and applications for brain-computer interfacing. In Human Interface and the Management of Information. Methods, Techniques and Tools in Information Design (pp. 705-714). Springer Berlin Heidelberg. http://dx.doi.org/10.1007/978-3-540-73345-4_80

Neuper, C., \& Pfurtscheller, G. (2001). Evidence for distinct beta resonance frequencies in human EEG related to specific sensorimotor cortical areas. Clinical Neurophysiology, 112(11), 2084-2097. http://dx.doi.org/10.1016/S1388-2457(01)00661-7

Niedermeyer, E., \& da Silva, F. H. L. (2005). Electroencephalography: Basic Principles, Clinical Applications, and Related Fields, Lippincott Williams \& Wilkins. Retrieved from http://books.google.jo/books?id=tndqYGPHQdEC

Oliver, J., Shantha Selva Kumari, R., \& Sadasivam, V. (2005). Wavelets for improving spectral efficiency in a digital communication system. Sixth International Conference on Computational Intelligence and Multimedia Applications, Las Vegas, Nevada: 198-203. http://dx.doi.org/10.1109/ICCIMA.2005.60

Pfurtscheller, G., Neuper, C., Flotzinger, D., \& Pregenzer, M. (1997). EEG-based discrimination between imagination of right and left hand movement. Electroencephalography and Clinical Neurophysiology, 103(6), 642-651. http://dx.doi.org/10.1016/S0013-4694(97)00080-1

Phinyomark, A., Limsakul, C., \& Phukpattaranont, P. (2010). Optimal Wavelet Functions in Wavelet Denoising for Multifunction Myoelectric Control. ECTI Transactions on Electrical Eng., Electronics, and Communications, 8(1), 43-52.

Phinyomark, A., Limsakul, C., \& Phukpattaranont, P. (2011). Application of Wavelet Analysis in EMG Feature Extraction for Pattern Classification. Measurement Science Review, 11(2), 45-52. http://dx.doi.org/10.2478/ 
v10048-011-0009-y

Phinyomark, A., Nuidod, A., Phukpattaranont, P., \& Limsakul, C. (2012). Feature Extraction and Reduction of Wavelet Transform Coefficients for EMG Pattern Classification. Elektronika ir Elektrotechnika (Electronics and Electrical Engineering), 122(6), 27-32. http://dx.doi.org/10.5755/j01.eee.122.6.1816

Phinyomark, A., Quaine, F., Laurillau, Y., Thongpanja, S., Limsakul, C., \& Phukpattaranont, P. (2013). EMG Amplitude Estimators Based on Probability Distribution for Muscle-Computer Interface. Fluctuation and Noise Letters, 12(3), 1350016. http://dx.doi.org/ 10.1142/S0219477513500168

Qahwaji, R., Colak, T., Al-Omari, M., \& Ipson, S. (2008). Automated Prediction of CMEs Using Machine Learning of CME-Flare Associations. Sol. Phys., 248(2), 471-483. http://dx.doi.org/10.1007/s11207-007-9108-1

Qingyang, L., \& Zhe, S. (2012). Method of Harmonic Detection Based On the Wavelet Transform. International Conference on Information and Computer Applications (ICICA), Hong Kong (pp. 213-217). http://dx.doi.org/10.4028/www.scientific.net/AMR.748.646

Riouel, O., \& Vetterli, M. (1991). Wavelet and signal processing. IEEE SP Mag., 8, 14-38. http://dx.doi.org/10.1109/79.91217

Romo-Vazquez, R., Ranta, R., Louis-Dorr, V., \& Maquin, D. (2007). EEG Ocular Artefacts and Noise Removal. 29th Annual International Conference of the IEEE Engineering in Medicine and Biology Society, EMBS 2007 (pp. 5445-5448). Lyon. http://dx.doi.org/10.1109/IEMBS.2007.4353577

Schalk, G., McFarland, D. J., Hinterberger, T., Birbaumer, N., \& Wolpaw, J. R. (2004). BCI2000: a general-purpose brain-computer interface (BCI) system. IEEE Transactions on Biomedical Engineering 51(6), 1034-1043. http://dx.doi.org/10.1109/ TBME.2004.827072

Selim, A. E., Wahed, M. A., \& Kadah, Y. M. (2008). Machine Learning Methodologies in Brain-Computer Interface Systems. Biomedical Engineering Conference, 2008. CIBEC 2008 (pp. 1-5). Cairo International. http://dx.doi.org/10.1109/cibec.2008.4786106

Sepulveda, F. (2011). Brain-Actuated Control of Robot Navigation. Advances in Robot Navigation. A. Barrera, InTech. http://dx.doi.org/10.5772/17401

Silverman, B. (2000). Wavelets in Statistics: Beyond the Standard Assumptions. Wavelet: The key to intermittent information (pp. 71-86). Oxford, New York: Oxford University Press. Retrieved from http://www.stats.ox.ac.uk/ silverma/pdf/rspaper.pdf

Sleight, J., Pillai, P., \& Mohan, S. (2009) Classification of Executed and Imagined Motor Movement EEG Signals. University of Michigan. Retrieved from http://www.scribd.com/doc/82045737/ICA

Su, Y., Qi, Y., Luo, J. X., Wu, B., Yang, F., Li, Y., ... Chen, W. D. (2011). A hybrid brain-computer interface control strategy in a virtual environment. Journal of Zhejiang University SCIENCE C, 12(5), 351-361. http://dx.doi.org/10.1631/jzus.C1000208

Tolić, M., \& Jović, F. (2013). Classification of Wavelet Transformed EEG Signals with Neural Network for Imagined Mental and Motor Tasks. International Journal of Fundamental and Applied Kinesiology, 45(1), 130-138. http://hrcak.srce.hr/file/153746

Tuntisak, S., \& Premrudeepreechacharn, S. (2007). Harmonic Detection in Distribution Systems Using Wavelet Transform and Support Vector Machine. 2007 IEEE Lausanne Power Tech, Lausanne (pp. 1540-1545). http://dx.doi.org/10.1109/PCT.2007.4538544

Vallabhaneni, A., Wang, T., \& He, B. (2005). Brain-Computer Interface. Neural Engineering (pp. 85-121). Springer US. http://dx.doi.org/10.1007/0-306-48610-5_3

Vidal, J. J. (1973). Toward Direct Brain-Computer Communication. Annual Review of Biophysics and Bioengineering, 2(1), 157-180. http://dx.doi.org/10.1146/annurev.bb.02.060173.001105

Wang, Y., Hong, B., Gao, X., \& Gao, S. (2007). Implementation of a Brain-Computer Interface Based on Three States of Motor Imagery. 29th Annual International Conference of the IEEE Engineering in Medicine and Biology Society, 2007 (pp. 5059-5062). EMBS 2007. http://dx.doi.org/10.1109/iembs.2007.4353477

Wolpaw, J. R., Birbaumer, N., McFarland, D. J., Pfurtscheller, G., \& Vaughan, T. M. (2002). Brain-computer interfaces for communication and control. Clinical Neurophysiology, 113(6), 767-791. http://dx.doi.org/10.1016/S1388-2457(02)00057-3 


\section{Copyrights}

Copyright for this article is retained by the author(s), with first publication rights granted to the journal.

This is an open-access article distributed under the terms and conditions of the Creative Commons Attribution license (http://creativecommons.org/licenses/by/3.0/). 